University of Nebraska - Lincoln

DigitalCommons@University of Nebraska - Lincoln

Journal for the Advancement of Developing

Economies

Economics Department

2016

\title{
Mobile Facial Recognition System for Patient Identification in Medical Emergencies for Developing Economies
}

Kingsley C. Nwosu

Saint Leo University

Follow this and additional works at: https://digitalcommons.unl.edu/jade

Part of the Econometrics Commons, Growth and Development Commons, International Economics Commons, Political Economy Commons, Public Economics Commons, and the Regional Economics Commons

Nwosu, Kingsley C., "Mobile Facial Recognition System for Patient Identification in Medical Emergencies for Developing Economies" (2016). Journal for the Advancement of Developing Economies. 10.

https://digitalcommons.unl.edu/jade/10

This Article is brought to you for free and open access by the Economics Department at DigitalCommons@University of Nebraska - Lincoln. It has been accepted for inclusion in Journal for the Advancement of Developing Economies by an authorized administrator of DigitalCommons@University of Nebraska - Lincoln. 


\title{
Mobile Facial Recognition System for Patient Identification in Medical Emergencies for Developing Economies
}

\author{
Kingsley C. Nwosu \\ Saint Leo University, Virginia, USA
}

\begin{abstract}
Medical emergencies are part of the common daily lives of people in developing and underdeveloped economies. Frequently, some of these medical emergencies end up tragically for many people in these countries due to many reasons, among which is the delivery of medical treatment when the patient is uncommunicative or unresponsive. The ability of the attending medical personnel to access a patient's medical history is critical for the quality of the treatment rendered. Unfortunately, today many lives are lost in low income economies during medical emergencies due to lack or inaccessibility of a patient's medical information. One of the major contributing factors of this paucity in records is attributable to the absence of reliable and cost-efficient healthcare delivery systems that support patient identification and verification. Due to the current ubiquity of mobile devices with their concomitant digital cameras, this paper explores the feasibility and practicability of using mobile platform and facial recognition technology as a means to deploying a cost-efficient system for reliable patient identification and verification.
\end{abstract}

Keywords: medical emergencies, developing economies, patient identification, facial recognition.

\section{INTRODUCTION}

In spite of the global efforts by healthcare providers to focus on preventive healthcare systems, medical emergencies continue to claim many lives in low income economies due to many reasons, including, but not limited to, insufficiencies of qualified medical personnel, unavailability of appropriate medical equipment, cultural barriers, cost, and unreliable delivery systems. Lack of stable, supportive computer networks makes it impossible for data sharing, and helps to compound the problem. Other contributing factors, as identified by Robertson, et al. (2009), include economic and geopolitical constraints, transportation, and geographic barriers. Medical emergencies are usually handled in three phases - at point of occurrence, during transportation, and at a health facility. Razum \& Kelly (2005), in their works in Zimbabwe, concluded the fate of an emergency patient depends greatly on what happens during the first phase of the treatment. The issues and problems surrounding the insufficiencies of medical personnel and unavailability of equipment have been amply discussed in Roudsariemail (2005); Conrad \& Gallagher (2015); Razzak et al. (2008); Chandran \& Lyn (2008); Scott et al. (2008); Kinfu et al. (2009); and Naicker et al. (2009). The focus of this paper is on the issues contributing to the unreliability of the emergency healthcare delivery process.

Under normal healthcare delivery process, the quality of the services rendered is greatly impacted by the knowledge and/or accessibility of a patient's medical information. Many healthcare delivery 
accidents have happened, in part, due to the absence or inaccessibility of a patient's medical history, as evidenced in the works by Castrejón, McCollum, Tanriover, \& Pincus, (2012; Tsukamoto (2012); and Grif (2011). This situation is complicated in a medical emergency when a patient is uncommunicative or unresponsive, and therefore unable to provide some medical information. What is needed to effectively address this problem is a system that can securely and reliably capture, store, and retrieve a patient's relevant medical information. The reliability depends greatly on how to verify or identify a patient during a medical emergency described earlier. Low income economies are notorious for the absence of healthcare infrastructure that facilities storage and access of patients' necessary medical information.

This system is an attempt to design and develop a cost-efficient and reliable system and platform to facilitate the capture, storage, access, and retrieval of patients' critical medical information for medical emergencies in developing and under-developed economies.

\section{GENERAL SYSTEM DESCRIPTION}

This system comprises a front-end for patient registration or enrollment; mobile application for patient identification, and a back-end for data storage and retrieval. The patient registration or enrollment can be done either by the patient's Primary Care Physician (PCP) with the authorization of the patient, or by the patient using a desktop or mobile application. Only critical medical information necessary for emergency medical care is captured during the enrollment, including the patient's passport-sized digital image. All textual information is stored in the back-end in encrypted form. Only authorized persons, who have successfully authenticated biometrically, are allowed access via the front-end mobile application.

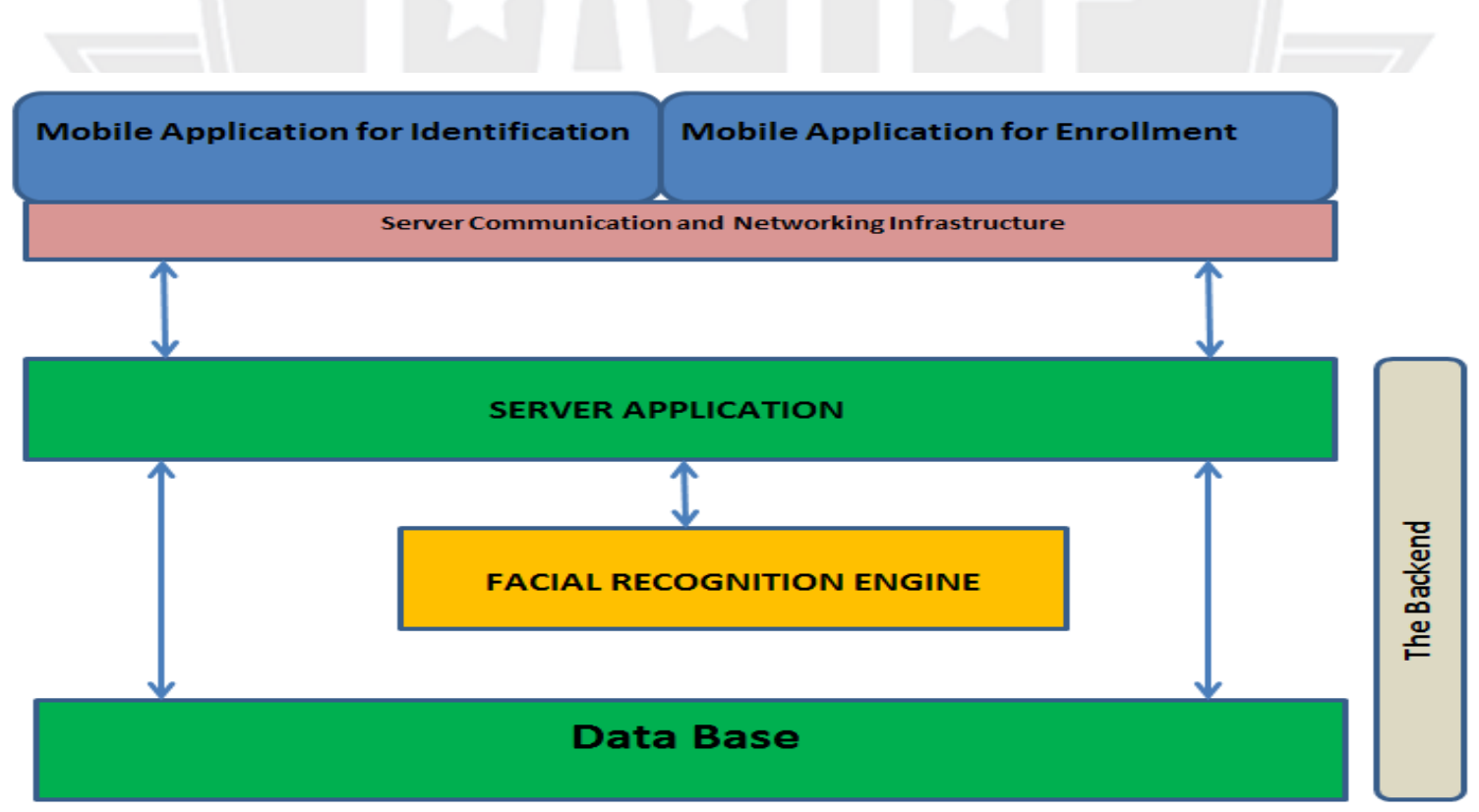

Fig. 1: General conceptual architecture of the system 


\section{The Front-end Applications}

The system's front-end comprises two mobile applications: (1) Patient Enrollment and (2) Patient Identification.

\section{Patient Enrollment Application (PEA)}

The PEA is used to enroll a patient in the system. The enrollment process involves the capture of necessary, critical emergency medical information, including the patient's passport-sized digital photograph. One component of this critical emergency medical information is the patient's list of allergies. There are different types of allergies which include, but are not limited to: Cockroach Allergy, Drug Allergies, Dust Allergy, Eye Allergies, Food Allergies, Insect Sting Allergies, Latex Allergy, Mold Allergy, Pet Allergies, Rhinitis, Sinusitis, and Skin Allergies. In addition to the allergies, the enrollment process captures some personal information about the patient such as the name, age, and gender, which are vital during the identification process. Furthermore, other optional pieces of information can be captured, such as the patient's past medical history, past surgical history, social history, family history, and medications. The patient enrollment can be done either by the patient's Primary Care Physician (PCP), with the authorization of the patient, or done directly by the patient. When using a mobile phone for registration, some of the enroller's personal information are automatically retrieved from the associated mobile phone and stored for security purposes and post enrollment validation. Distinction is made between information obtained via enrollment from a patient and the PCP. For the purposes of quality and reliability of patient information, a distinction is made about the sources of the information both at storage and retrieval when applicable information is accessed. This helps to convey a degree of confidence about patient information based on the sources of the information. For security and privacy purposes, all original textual data, without privacy implications, and that will never be part of the identification criteria, are stored in encrypted form.

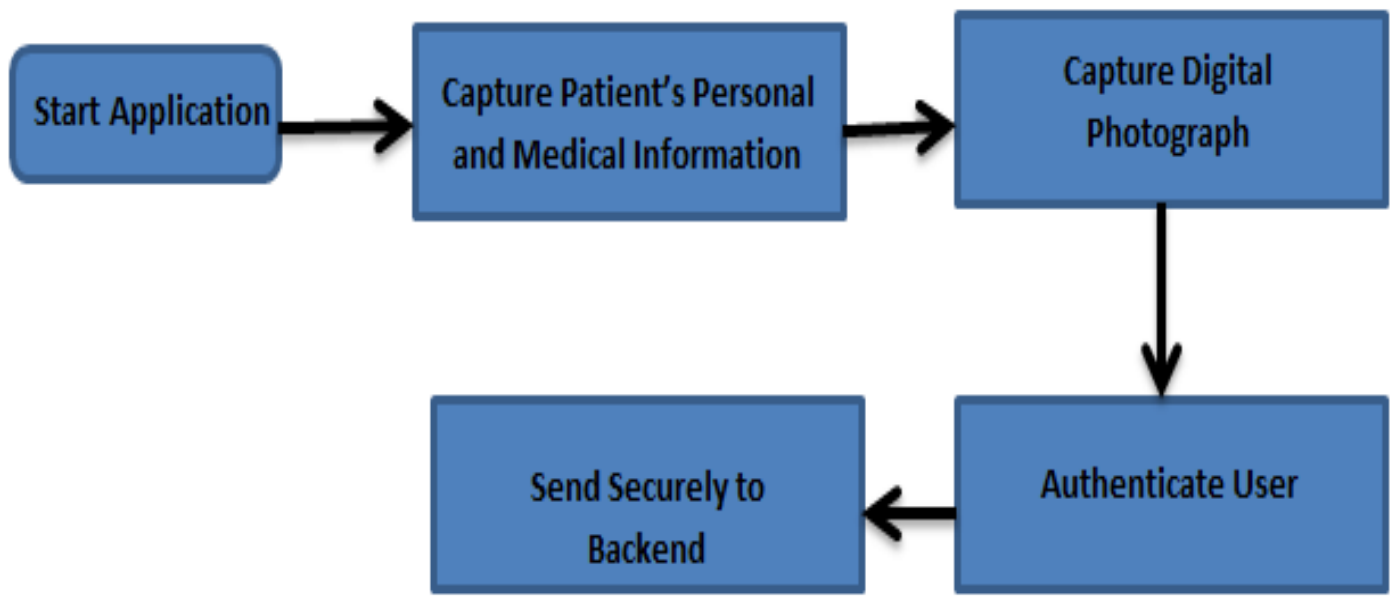

Fig. 2: Patient enrollment flow diagram 


\section{Patient Identification Application (PIA)}

The other component of the front-end is the Patient Identification Application which is used to identify a patient during an emergency. In the best-case scenario during a medical emergency, the patient's name, age, and gender are known. In that case, a verification operation is performed with the age, name, and gender to retrieve the associated information, if available. In the worst-case scenario, neither the name nor age is known. In this case, an identification operation is required as described below, (see "System's Facial Recognition Technique") using the patient's gender and estimated age. Depending on the result of each matching request, the user can repeatedly modify or tune the patient's age and perform another matching request, as detailed in the matching technique below.

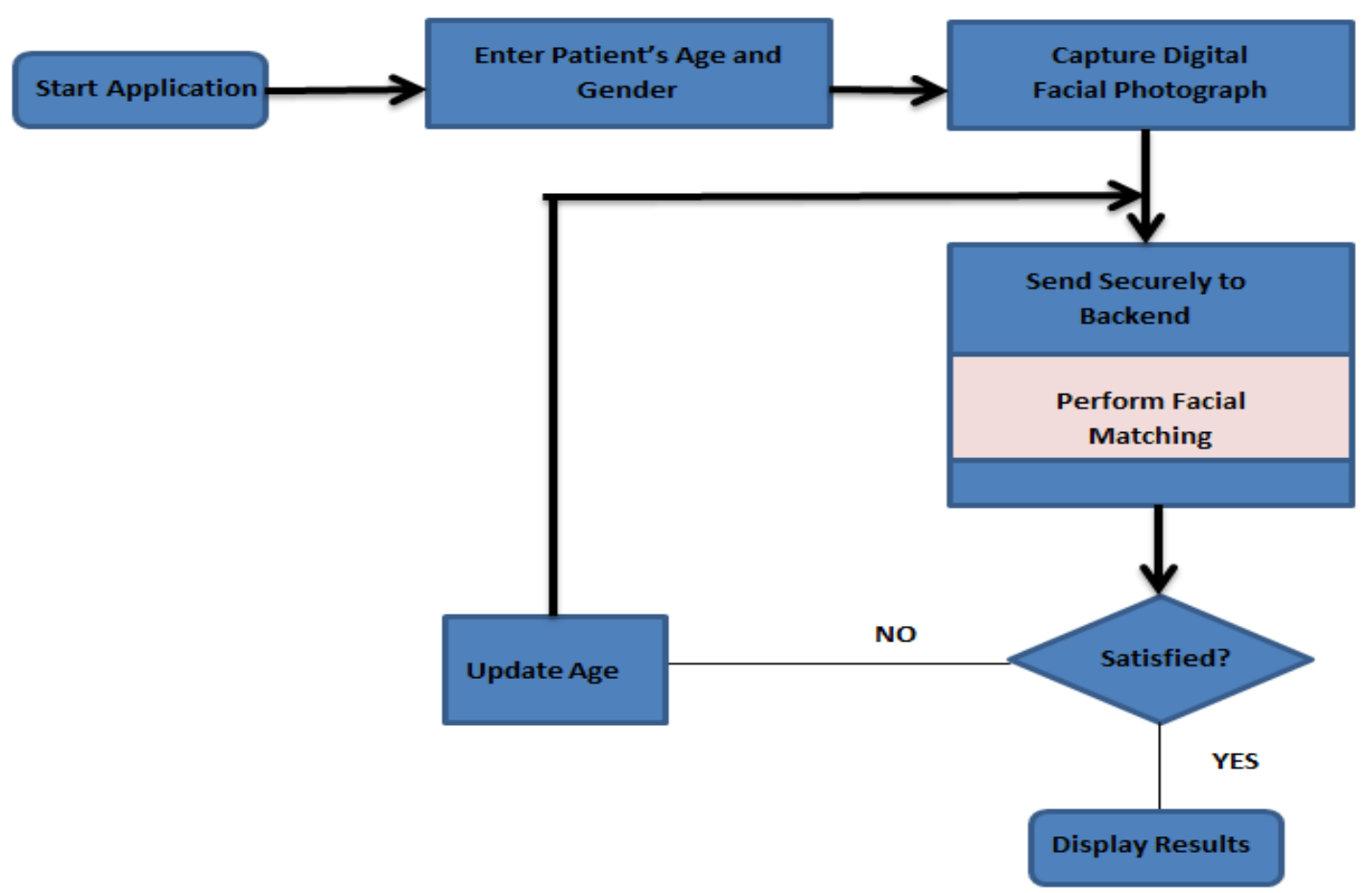

\section{Fig. 3: Patient identification flow diagram}

As detailed below, facial recognition technique is a similarity-based algorithm that determines the degree of similarity between two facial templates. The higher the degree of similarity, the more equal the two objects. As a result, the number of matches between one face and many faces depends on the similarity threshold specified. The similarity threshold is usually a number between 0.01 and 1.0 where a value of 1 indicates absolute similarity. Usually, a similarity threshold of $\mathrm{S}$ is expressed as $0<\mathrm{S}<=1.0$.

The task of identifying a patient from a database of patients is similar to the classical problem of searching in computing. How quickly one can find or identify an element from among many elements is a function of the size and nature of the elements. Over the years in the field of data structures and algorithms, many techniques and strategies have been designed and developed to 
optimize the search process. For multifarious or multi-component or structured elements, one of the effective strategies is determining the target set of elements from the complete set. By reducing the target set of elements, one is effectively reducing the effective search time. As a result, this system utilizes tunable parameters in the form of the patient's age and gender to reduce the effective search set.

\section{The Back End}

The system's back-end is responsible for performing the facial matching, encryption, decryption, storage, and retrieval. The back-end accepts the enrollment data from the front-end application, formats the data as required, performs encryption and facial template extraction, and stores the information in the database. The enrollment fails if the facial template extraction was unsuccessful. During patient identification, the back-end performs the facial matching activity and returns the matching patients' facial images and subsequent medical information. Whenever a backend request failed, it's the responsibility of the front-end applications to determine the next course of action whether to modify the request and repeat it or terminate the process.

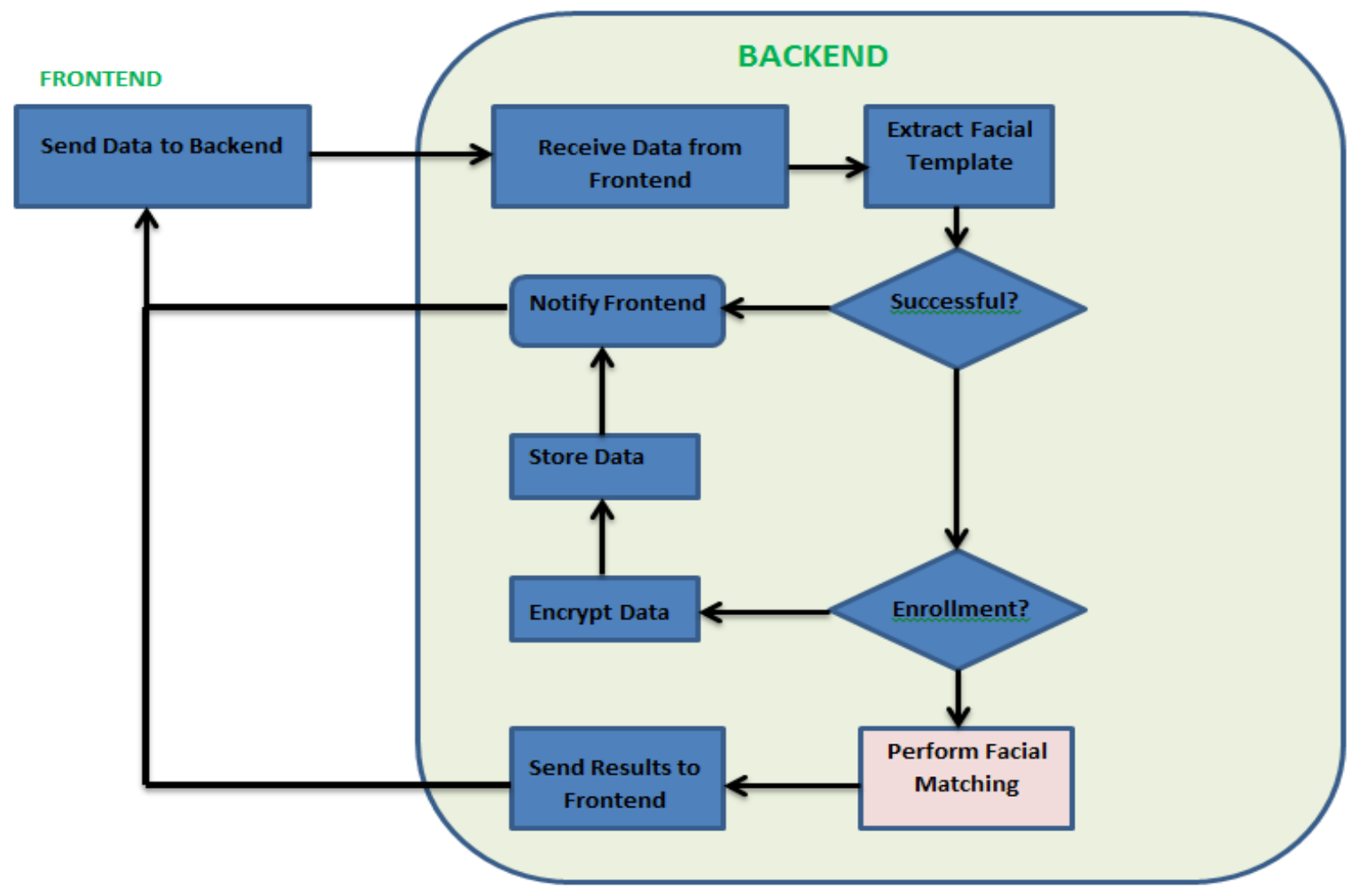

Fig. 4: Backend flow diagram

\section{The Facial Recognition Engine}

Biometrics verification and identification are the processes of using an individual's measurable physiological or behavioral attributes to either confirm or deny the stated identity of the individual or determine the identity of the individual. The physiological or behavioral attributes of an 
individual have to do with the measurable characteristics of the individual and related to the functioning of the body, such as fingerprints, face, DNA, Iris, Palm, voice, signature, keystroke dynamics, etc. Basically, physiological data has to do with data derived from the measurement of a part of a person's anatomy. Behavioral data has to do with data derived from measurement of an action performed by a person. Verification is the process of determining whether someone or something is, in fact, who or what it is declared to be. Identification is the process of establishing the identity of an individual. Verifying or confirming a person's identity can be accomplished by using something the person knows (password), something the person has (token), or something that's part of the person (biometrics). Collectively, they provide the highest degree of security; however, individually, biometrics has the highest degree of security and reliability. The use of password, token, biometrics, or combination thereof depends on the target objective of the system. Each mode of verification has its inherent problems and issues, such as recollection, management,

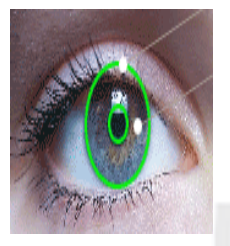

and intrusion.

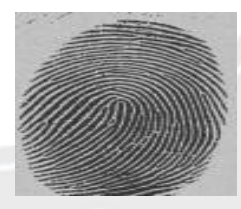

Why Facial Recognition?

As a biometric technique, facial recognition is the least intrusive of all the biometric technologies. Facial recognition systems can surreptitiously take a picture of a person's face when he/she is present within a defined area. The target object for facial identification may not consciously participate in the process, that is, he/she does not need to take any particular posture for the facial image to be captured. The camera can determine the presence of a face and capture the image.

The facial recognition software operates by detecting a face and then measuring the various features of the face. Everyone's face has several, distinguishable characteristics that constitute the facial features. These features include but are not limited to: distance between the eyes, width of the nose, depth of the eye sockets, the shape of the cheekbones, and the length of the jaw line. These features are then measured and aggregated to produce a quantifiable numerical code that is used in facial recognition process. The entire process involves the following steps:

- Detection/Capturing - An image can be obtained by scanning an existing photograph or using a manually controlled video camera or by a camera automatically detecting a face.

- Extraction/Representation - Unique data (code) is extracted from the sample and a template is created. This may involve using and comparing serial samples.

- Matching - The system then decides if the features extracted from the new sample are matching or not.

\section{The System's Facial Recognition Technique}


The facial matching (recognition) approach used in this system uses a patient's gender, estimated age, and photo image (live or stored) to retrieve a set of patients' facial templates that meet a given similarity threshold in comparison with the target patient. The age and similarity parameters can be adjusted during successive iterations of an identification session, in order to obtain an optimal result. A similarity value measures the degree of similarity between two facial objects. Therefore, a value of 1.0 indicates a $100 \%$ match between two facial objects. The lower the similarity value, the higher the probability that more facial objects will match the target object.

To describe the facial matching technique utilized in here, we let:

- $\quad \boldsymbol{a}$ represent the estimated age of a patient and $\mathbf{a}_{\mathbf{z}}$ represent the estimated age during the $\mathbf{z}^{\text {th }}$ iteration of a given identification session.

- $g$ represents the gender of the patient.

- $\boldsymbol{S}$ represent a set of similarity threshold values 0.1 to 1.0 with 0.1 differential, where $\boldsymbol{S}$ $=\{.1, .2, .3, \ldots, 1.0\}$ and $\mathbf{S}_{\mathbf{i}}$ represents the $\mathbf{i}^{\text {th }}$ element of $\boldsymbol{S}$; and $\mathbf{S}_{\text {sys }}$ represents the system default threshold value.

- $\quad \boldsymbol{F}\left(\mathbf{a}_{\mathrm{z}}, \boldsymbol{g}, \boldsymbol{j}\right)$ represent the face matching function that compares two face objects and returns a value $\boldsymbol{y}$ where $0<=\boldsymbol{y}<=1.0 ; \boldsymbol{j}$ represents any face object from the target database that meets the $\mathbf{a}_{\mathbf{z}}$ and $\boldsymbol{g}$ selection criteria.

- $\boldsymbol{H}$ represent the face matching process that uses $\boldsymbol{F}\left(\mathbf{a}_{\mathbf{z}}, \boldsymbol{g}, \boldsymbol{j}\right)$ and yields a set of facial objects, $\boldsymbol{\Omega}$.

- $\quad \boldsymbol{z} \boldsymbol{H}$ represent the $\mathbf{z}^{\text {th }}$ iteration within a given identification session where $\boldsymbol{z}>=0$.

- $\boldsymbol{z} \boldsymbol{H}\left(\boldsymbol{a l l}, \mathbf{S}_{\mathrm{i}}\right)$ represent the result of applying $\boldsymbol{S}_{\boldsymbol{i}}$ threshold value, that is, $\boldsymbol{F}\left(\mathbf{a}_{\mathrm{z}}, \boldsymbol{g}, \boldsymbol{j}\right)>=\mathbf{S}_{\mathrm{i}}$.

- $\quad \boldsymbol{z} H\left(\max , S_{\mathrm{i}}\right)$ represent the result of applying $S_{\mathrm{i}}$ threshold value such that there exists

- $\quad F\left(\mathrm{a}_{\mathrm{z}}, g, j\right)=\max$ and for all $m !=j, F\left(\mathrm{a}_{\mathrm{z}}, g, m\right)<=\max$.

Therefore, for a given $\mathbf{t}^{\text {th }}$ iteration within an identification session,

$$
\begin{aligned}
& \Omega=t H\left(\text { all, } S_{\text {sys }}\right) U t H\left(\max , S_{\text {sys }}\right) \quad \text { if } t=0 \\
& \Omega=t H\left(\text { all, }, S_{i}\right) \text { if } t>0
\end{aligned}
$$

Consequently, the associated pseudo code for the $\mathbf{z}^{\text {th }}$ iteration for an identification session is thus:

\title{
3 PROCEDURE
}

\author{
$G\left(\mathbf{a}_{\mathbf{z}}, \mathbf{g}, \mathbf{S}_{\mathbf{i}}\right)$ \\ Initialize $\boldsymbol{z} \boldsymbol{H}\left(\boldsymbol{m a x}, \mathbf{S}_{\mathbf{i}}\right)$ to empty \\ Obtain $\Omega$ for $\boldsymbol{F}\left(\mathbf{a}_{\mathbf{z}}, \mathbf{g}, \boldsymbol{j}\right)$ \\ For each face template in $\boldsymbol{\Omega}$
}

Compute the similarity value

If similarity value $>=\mathbf{S}_{\mathbf{i}}$ then

End if

Add face template ID to $\mathbf{z} \boldsymbol{H}\left(\boldsymbol{a l l}, \mathbf{S}_{\mathbf{i}}\right)$

If similarity value $>$ similarity value of $\boldsymbol{H}\left(\boldsymbol{m a x}, \mathbf{S}_{\mathbf{i}}\right)$ then

Set $\boldsymbol{z} \boldsymbol{H}\left(\boldsymbol{m a x}, \mathbf{S}_{\mathbf{i}}\right)$ to current face ID 


\author{
End if \\ End For each \\ If $z==0$ then \\ End if \\ Add $z \mathbf{H}\left(\max , \mathbf{S}_{\mathbf{i}}\right)$ to $z \mathbf{H}\left(\mathbf{a l l}, \mathbf{S}_{\mathbf{i}}\right)$ \\ Return $\boldsymbol{z} \boldsymbol{H}\left(\right.$ all, $\left.\mathbf{S}_{\mathrm{i}}\right)$ \\ End PROCEDURE
}

\title{
4 INTEGRATION WITH GOOGLE GLASS
}

Google glass is a network-enabled, eyeglass-like headset that displays information like a smartphone. It was developed by Google X, a division of Google. It responds to user's voice recognition, manual commands, and eye movements. It consists of a touchpad for manual control of the device; a high-definition camera for taking pictures and videos; and a liquid crystal-based display.
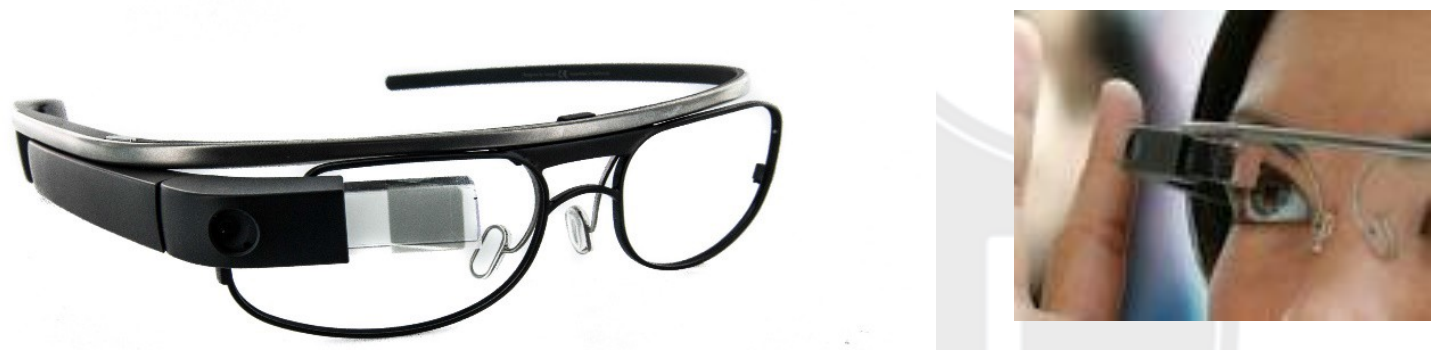

There have been documented uses of Google glass in the healthcare delivery such as (Greenfield, 2015) to access patient records, check live patient vitals, and physicians' collaboration; (Farr, 2014) a cloud-based patient/physician medical record sharing environment; and (Metz, 2015) that enables live-streaming of patients' visit thereby eliminating the need for Electronic Health Records. Google glass will be integrated with this system in order to leverage the ease-of-use that it provides for patient facial recognition. This integration is seamless given that Google glass is also an Android-based device.

\section{LIMITATIONS AND CHALLENGES}

One of the major assumptions of this system is the availability of patient registration, that is, the willingness and cooperation of the patients and their healthcare professionals to register with the system and provide the necessary medical information. Due to lack of evidential benefits from documentation exercises, many people in low income economies are usually not motivated with the idea of either participating with such endeavors, and/or providing reliable information. As such, necessary advocacy and enlightenment may be necessary to educate and inform the target population about the benefits of the system.

\section{SYSTEM STATUS AND TESTING RESULTS}

The front-end of this system has been designed and partially implemented. The PEA and PIE have been prototyped as Java applications for testing and demonstration purposes. The back-end has been fully implemented. The test database was populated with one to three thousand facial templates with 1:1 male/female ratio. 
Due to the fact that an identification process may be time-intensive because of its $\mathbf{O}(\mathbf{N})^{1}$ execution time when matching $\boldsymbol{N}$ facial templates, it's always a concern in any facial matching system to determine the performance efficiency of the system. Our evaluation environment used a LINUX 32-bit machine with Apache Web Server, MySQL 5.x Database Server, two 2.4 GHz Processors and 4 GB RAM. The CPU matching time for 1,000 to 3,000 facial templates in the database with a percentage hit of $30 \%-50 \%$ ranged from 7.5 milliseconds to 38.3 milliseconds. The total CPU time to retrieve all the applicable facial templates and IDs from the database ranged from 32.0 milliseconds to 163.0 milliseconds.

This system is still in-progress and we are planning to complete the application development, system integration, and system testing using our Computer Technology Excellence Laboratory (CTEL) which is currently under development.

\section{CONCLUSIONS}

This paper attempts to address the issue of patient identification during a medical emergency when a patient's medical information may become inaccessible as a result of the patient's inability to communicate effectively in order to provide the needed medical information. The system comprises two front-end mobile applications that are used to enroll and identify a patient based on the patient's facial recognition, in conjunction with the gender and tunable age parameters. This system utilizes efficient and effective facial template management and matching technique to determine whether a given patient already exists in the database, and to retrieve the associated medical information. The ability to quickly identify a patient, even when the patient is unresponsive, enables healthcare providers to access a patient's medical history which is invaluable for quality of care.

\section{REFERENCES}

Bulletin of the World Health Organization: the International Journal of Public Health; 80(11). (pp. 900-905).

Castrejón, I., McCollum, L., Tanriover, MD, \& Pincus, T. (2012). Importance of Patient History and Physical Examination in Rheumatoid Arthritis Compared to Other Chronic Diseases: Results of a Physician Survey. Arthritis Care \& Research, Vol. 64, No. 8, August 2012 (pp. $1250-1255$ ).

Chandran, K. \& Lyn, T. E. (2008, October 1). Lack of medical workers causes new health crisis in developing countries. New York Times (Asia Pacific).

Farr, C., (2014). Startup launches 'first wearable health record' for Google Glass, Reuters, June 2014.

Greenfield, R. (2015, January 2015). Google Glass Is Not Dead Yet: Augmedix, A Glass for Doctors Startup, Raises \$16 Million. Retrieved from http://www.fastcompany.com/.

Grif Alspach, J. (Editor) (2011). The Importance of Family Health History: Your Patients' and Your Own. Critical Care Nurse, 2011 vol. 31 no. 1 (pp. 10-15).

Kinfu, Y. et al (2009). The health worker shortage in Africa: are enough physicians and nurses being trained? Bulletin of the World Health Organization 2009 (pp. 225-230).

Metz, R. (2015, July 2015). Google Glass Finds a Second Act at Work. MIT Technology Review.

\footnotetext{
${ }^{1} \mathrm{Big} \mathrm{O}$ notation is used to describe the performance or complexity of an algorithm. It's mostly used to describe the worst-case scenario of an algorithm either in the execution time or space used.
} 
Naicker, S. et al (2009). Shortage of healthcare workers in developing countries-Africa. Ethnicity \& Disease 02/2009; 19(1 Suppl 1): S1-60-4.

P. Conrad, P. \& Gallagher, E. B. (2015). Health and health care in developing countries: Sociological perspectives. ISBN: 978-1-56639-027-9.

Razum, O., \& Keller, P. A. (2002). Emergency medical care in developing countries: is it worthwhile?

Razzak, J. A. et al (2008). Assessing emergency medical care in low income countries: A pilot study from Pakistan. BMC Emergency Medicine 2008, Vol. 8, No. 8. Retrieved from http://www.biomedcentral.com/1471-227X/8/8

Robertson, J., DeHart, D., Tolle, K., Heckerman, D., Healthcare Delivery in Developing Countries: Challenges and Potential Solutions. The Fourth Paradigm, Microsoft Research, 2009.

Roudsariemail, B. S. et al (2007). Emergency Medical Service (EMS) systems in developed and developing countries. International Journal of the Care of the Injured, Volume 38, Issue 9 (pp. 1001-1013).

Scott, M. L. et al (2004). Brain drain or ethical recruitment? Medical Journal of Australia, 2004; 180 (4): (pp 174-176).

Tsukamoto, T. (2012). The contribution of the medical history for the diagnosis of simulated cases by medical students. International Journal of Medical Education. 2012; Vol. 3 (pp. 78-82). 